\title{
The Modal Characteristics of Circular Vibrating Screen and Kinematics Analysis
}

\author{
Yijun Zhou ${ }^{\mathrm{a}}$, Xiaodong Zheng ${ }^{\mathrm{b}}$, Lei Zhang ${ }^{\mathrm{c}}$
}

School of Mechanical Engineering, Anhui University of Science and Technology, HuaiNan, 232001, China

azhy31130@163.com, ${ }^{\mathrm{Pb}}$ 1361798398@qq.com, ${ }^{\mathrm{Pc}}$ 1243286532@qq.com

Keywords:Modal analysis; Natural frequency; Kinematics simulation.

\begin{abstract}
The virtual prototype of circular vibrating screen was Established, and the overall structure and working principle of the device were described in detail.The natural frequency of circular vibrating screen was obtained by using finite element analysis software ANSYS to modal analysis,which can provide theoretical basis for the design of vibration frequency and avoid resonance, while using ADAMS software to simulation of the model and analysis to achieve ideal screening effect rotation speed, which can provide reference and basis for the follow-up design and product improvement, and also has a reference value to other vibration machinery research.
\end{abstract}

\section{Introduction}

Circular vibrating screen is a kind of mechanical equipment, which is based on vibration principle. It is used to have a primary screening machinery mainly for the raw material such as coal mines. Because of the bad working environment for a long time, so not only should ensure that the screening machine of the normal screening effect but also the reliability of the equipment structure, working life, environmental protection should meet the basic requirements. At present, the structure of the vibration equipment is not perfect, there is a short life, the screening effect is not ideal, the use of noise and other issues in the actual production process[1]. In this paper, the dynamic and kinematic analysis method is used to establish the three-dimensional model of the 2YAH1548 type circular vibrating screen of the $7.2 \mathrm{~m}^{2}$, and the dynamic performance analysis and kinematic simulation analysis are carried out.

\section{Working principle of circular vibrating screen}

The circular vibrating screen is used for coal preparation and final screening (grading), The main part of the screen are:box, vibrator, vibration isolation device, screen, fastening device, transmission device and a supporting device [2]. The mechanism as shown in Figure 1. The circular vibrating screen is characterized by the whole welding and firm and solid. The main shaft of the exciter is equipped with an eccentric shaft, and the excited vibration parts are arranged by the motor, the triangle belt, the bearing block, the eccentric block and so on. Vibrator is driven by motor, so that the transmission shaft is rotate at a certain speed and the inertia force of the eccentric block and the eccentric mass produce the circular motion of the sieve box. The screen box is provided with a damping spring support member, to reduce the amplitude and oscillating sieve.

Circular vibrating screen is widely used in $25 \mathrm{~mm}$ above the coarse grain size sieving, generally more than $100 \mathrm{t} / \mathrm{h}$ for processing capacity of the following occasions. The dip angle of the screen is generally $15-20$, but for the more difficult screening or large materials abrasion we have to choose a relatively large screen angle [4] point. In this paper, the angle of the screen surface of the circular vibrating screen is 20 . 

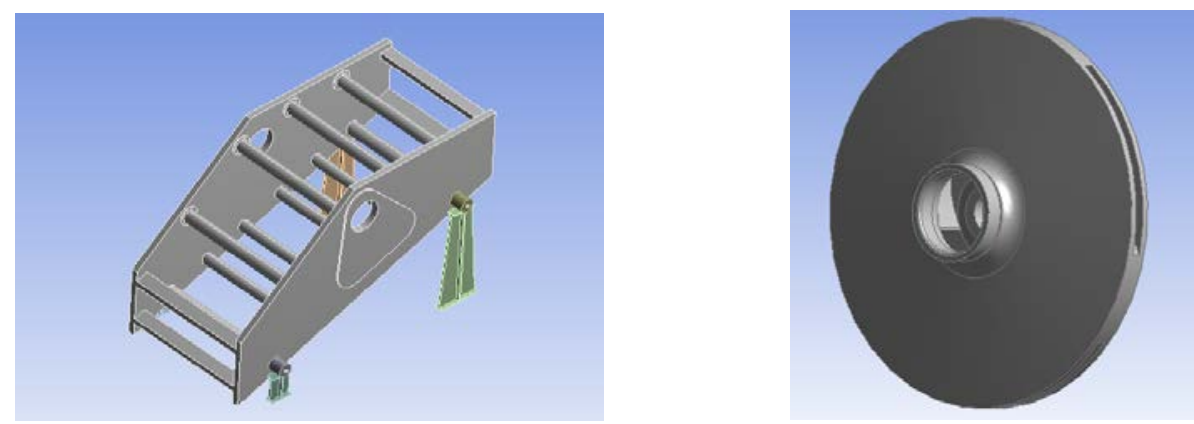

Fig. 1 The three-dimensional model of the circular vibrating screen mechanism

\section{Modal analysis of circular vibrating screen}

According to the model of the circular vibrating screen and the related parameters, the values of the parameters are shown in Table 1.

Table 1 Parameters of 2YA1548 circular vibrating screen [5]

\begin{tabular}{|c|c|c|c|}
\hline designation & value & designation & value \\
\hline Length of screen box & $4904 \mathrm{~mm}$ & $\begin{array}{l}\text { Quality of screen } \\
\text { box }\end{array}$ & $7404 \mathrm{~kg}$ \\
\hline Wide of screen box & $2715 \mathrm{~mm}$ & Height of screen box & $2943 \mathrm{~mm}$ \\
\hline $\begin{array}{l}\text { Amplitude of screen } \\
\text { box }\end{array}$ & $5.5 \mathrm{~mm}$ & Screen frequency & 755rpm \\
\hline Area of screen surface & $7.2 \mathrm{~m} 2$ & Double amplitude & 2 \\
\hline Screen length & $4.8 \mathrm{~m}$ & Screen width & $1.5 \mathrm{~m}$ \\
\hline Intensity of vibration & 4 & $\begin{array}{l}\text { The strength of the } \\
\text { projectile }\end{array}$ & 4 \\
\hline Dip angle of screen & $20^{\circ}$ & $\begin{array}{l}\text { Direction of } \\
\text { vibration }\end{array}$ & $45^{\circ}$ \\
\hline Vibrator type & YA110 & Double amplitude & 11 \\
\hline Amount of treatment & 50t/h.m2 & Speed of material & $0.034 \mathrm{~m} / \mathrm{s}$ \\
\hline
\end{tabular}

\section{The establishment of the screen box}

The screen box was made of a block of steel plate welded, the plate material was the same, but the thickness was not unique and it's kinds of thickness was not much. The side plate is connected by high strength bolts, which can be regarded as a whole [6]. So the sieve box was all the use of shell element in the establishment of the finite element model. But in the finite element mesh, the nodes of the connecting elements of the different parts must be noted to ensure that the load can pass [7], otherwise, there will be a discrete phenomenon in the design of the parts , which leads to the calculation of the result be not accurate or calculation error. In this paper, the three-dimensional model was imported into the finite element software ANSYS, and the modal analysis of the screen box was carried out.

\section{Simulation of screen box}

In the actual production, the circular vibrating screen is generally supported by 8 groups of four rubber springs .Spring and the spring supporting beam on the screen box are fixed together by [8]. In this paper, the spring damping element in ANSYS was used to simulate the 8 rubber springs. In the ANSYS analysis, except for the box with the axial restraint,but also Plus the size of the axial stiffness of the 1/3 lateral stiffness in The lateral stiffness since the movement direction of the sieve box was not fixed. One end of the lateral stiffness spring was connected with the joint of the spring supported beam, and the other end was fixed with a fixed connection mode, which was the same as the axial stiffness of the spring. In the past, the constraint of the rubber spring was set in the $X$ and $\mathrm{Y}$ direction respectively, and the $\mathrm{Z}$ direction was set at all nodes, this method can cause the natural frequency of the circular vibrating screen to be much greater than the value of its working frequency. So this paper used three directions of the simulation of the rubber spring, and choosing 
the stiffness of the spring reasonably through the understanding of the rubber spring performance. Figure 2 for the ANSYS software simulation of rubber spring. In this paper, the sieve box of circular vibrating screen was defined by the shell element, and we chose the shell63 element in the end because the number of shell element is more.Using the mass 21 point mass unit to replace the unbalance mass of the eccentric wheel. The mass 21 element is expressed by the point element, Its space six degrees of freedom were $\mathrm{x}, \mathrm{y}, \mathrm{z}$ axis of rotation and $\mathrm{x}, \mathrm{y}, \mathrm{z}$ direction linear displacement, respectively, each freedom degree of inertia and quality [27]. The spring damping element was used in the most simple spring element -combin14 unit, which can define the effect of the longitudinal torsion in two or three dimensions. Combin14 is no quality, so in the design, the quality of the spring does not need to consider.The solid element was the solid45 unit, which was used to define the spring supported beam, and the unit selection was shown in Table 2.

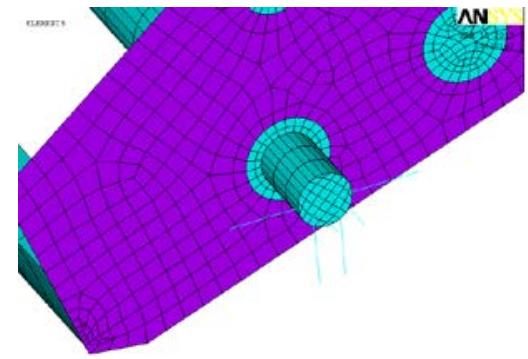

Fig. 2 Simulation of rubber spring

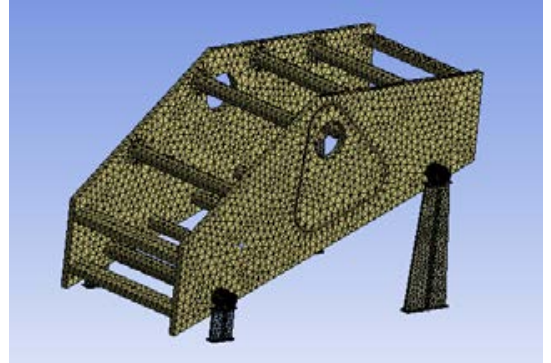

Fig.3 Grid division of circular vibrating screen

\section{Unit material selection}

In this paper, the sieve box of circular vibrating screen was defined by the shell element and as the number of shell element was more, so we chose shell63 unit. Using the mass21 point mass unit to replace the unbalance mass of the eccentric wheel. The mass21 element was expressed by the point element, and its space six degrees of freedom were $\mathrm{x}, \mathrm{y}, \mathrm{Z}$ axis of rotation and $\mathrm{y}, \mathrm{Z}, \mathrm{X}$ direction linear displacement, respectively, each freedom degree of inertia and quality [27]. The spring damper unit used the simplest spring element combin14 element. The combin14 unit can define the effect of longitudinal or torsion in two or three dimensions. The quality of the spring don't need to be considered in the design since combin14 is no quality. The solid element was the solid45 unit, which was be used to define the spring supported beam, and the unit selection was shown in Table 2.

Table 2 unit and its real constant

\begin{tabular}{|c|c|c|c|c|c|c|}
\hline Order & Element & \multicolumn{5}{|c|}{ Real constant } \\
\hline \multirow[t]{2}{*}{1} & \multirow[t]{2}{*}{ shell63 } & \multicolumn{5}{|c|}{ Sickness of slab/m } \\
\hline & & 0.01 & 0.012 & 0.014 & 0.026 & 0.056 \\
\hline 2 & solid45 & & & & & \\
\hline 3 & mass21 & \multicolumn{5}{|c|}{$200 \mathrm{Kg}$} \\
\hline 4 & $\begin{array}{c}\text { combin1 } \\
4\end{array}$ & \multicolumn{2}{|c|}{$\begin{array}{l}\text { Longitudinal } \\
\text { stiffness } / N / m^{3} \\
\quad 4.8 * 105\end{array}$} & \multicolumn{3}{|c|}{$\begin{array}{c}\text { Lateral rigidity } /_{N / m^{3}} \\
1.6 * 105\end{array}$} \\
\hline
\end{tabular}

\section{Division of finite element mesh}

The intelligent partition of the hollow beam and the front and back baffle was made. When the grid was divided into a grid. We made the contact part of the crossbeam and the sieve plate for intelligent division, because these places were prone to the phenomenon of stress concentration. We put on both sides of the sieve size appropriate division according to the need, so that you can save the calculation time, but also to reduce the burden of the computer. The different parts of the circular vibrating screen were divided According to different conditions, and the corresponding constraints were set up, finally the generated 3D model is shown in Figure 3.

\section{Results and conclusions of modal analysis}

Carrying out modal analysis of the circular vibrating screen by using the finite element software ANSYS , then the modal frequency and vibration mode of the 1-15 mode can be obtained, see table 
3.

Table 3 natural frequencies of the order

\begin{tabular}{|c|c|c|c|c|c|}
\hline Modal order & 1 & 2 & 3 & 4 & 5 \\
\hline Modal frequency & 1.5505 & 1.5773 & 2.0468 & 2.2866 & 2.5436 \\
\hline Modal order & 6 & 7 & 8 & 9 & 10 \\
\hline Modal frequency & 2.687 & 30.705 & 39.101 & 50.743 & 60.305 \\
\hline Modal order & 11 & 12 & 13 & 14 & 15 \\
\hline Modal frequency & 60.305 & 65.722 & 84.194 & 85.534 & 85.582 \\
\hline
\end{tabular}

We obtained the conclusion that the first 6 order vibration sieve, sieve box is mainly along the $\mathrm{X}, \mathrm{y}, \mathrm{z}$ direction of the translation and torsional rotation from the analysis results. From the beginning of the seventh order, which was mainly elastic vibration, bending torsion between the sieve box side and bending deformation and beam. The sixth order natural frequency was $2.687 \mathrm{~Hz}$, and the seventh order natural frequency was $30.7 \mathrm{~Hz}$. We should try to avoid the natural frequency of each order to avoid resonance In the design of the excitation frequency,figure 6 was the 4 order mode of vibration and figure seventh was the 5 order mode of vibration .

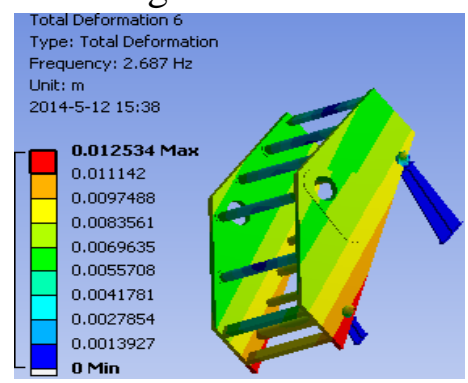

Fig.4 4 order vibration mode network

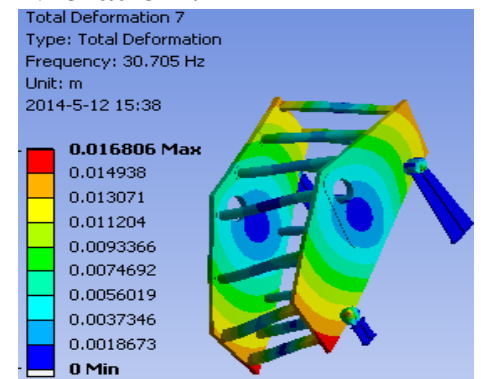

Fig.5 5 order vibration mode network

From table 3 it can be seen that the mode frequency was above $13 \%$ of the operating frequency, so it was far from the same frequency. That is, the design of the circular vibrating screen is reasonable.

\section{Analysis of ADAMS motion characteristics of circular vibrating screen}

We Studied the vibration effect through the kinematic simulation analysis of the circular vibrating screen with ADAMS.The three-dimensional model of circular vibrating screen was kept as Parasolid (*.x_t) format,and then integrated into ADAMS,Set the material properties of the individual components, the appearance of color, and added constraints. The model environment was set up with gravity, the ground and the support set,the screen box and the supporting body was arranged sleeve stress constraints and spring constraint, and between the sieve box and the exciter was provided with rotating sieve box side, $\mathrm{X}, \mathrm{Z}$ direction set sleeve force constraint. Figure 6 was the state after adding constraints.

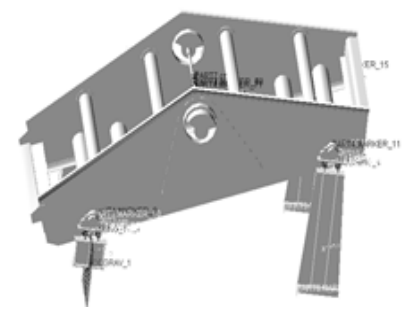

Fig.6 Adding constraints for 3D models

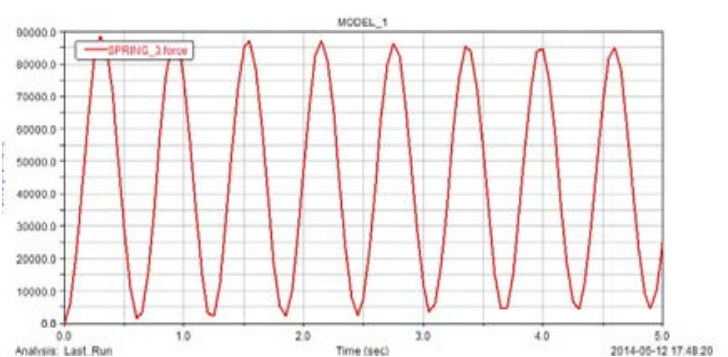

Fig.7 the relationship of the force and the time

Before simulation, the first was to determine the preload of the elastic element, we carried out the simulation of the whole virtual prototype in the non driving state. after the end, the preload size was obtained by means of an elastic element, in addition, the corresponding preload on the elastic element was added before the simulation. Since the spindle was rotated relative to the screen box, then a rotation drive was added to the main shaft, and the driving speed is set to $755 \mathrm{r} / \mathrm{min}$. The relationship between the force and time of the four support springs after applying the preload was 
shown in Fig.7.

Setting up the simulation time of 50s, the 1000 step simulation step and using ADAMS simulation to get the whole screen box in Z, Y direction of displacement, acceleration value. Figure 8. 9 showed the relationship between the displacement and the time of the screen box in the $\mathrm{Y}$ and $\mathrm{Z}$ directions, the relationship between the acceleration and time in the $\mathrm{Y}$ and $\mathrm{Z}$ directions of the screen box in 10 and 11, respectively. The lateral movement of the screen box was not studied.

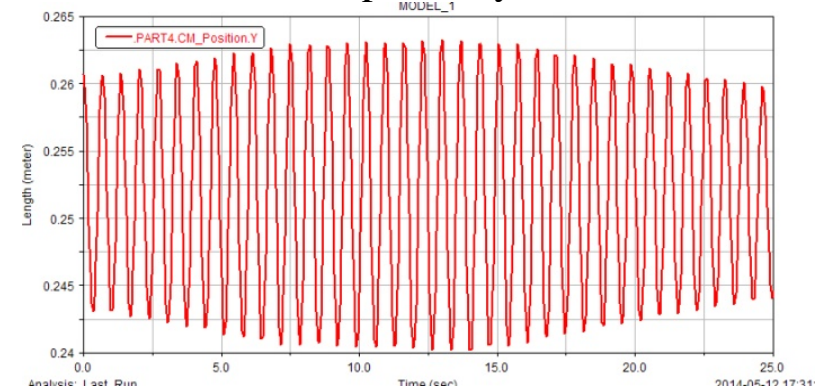

Fig. 8 the relationship between the displacement and the time of the screen box in the Y direction

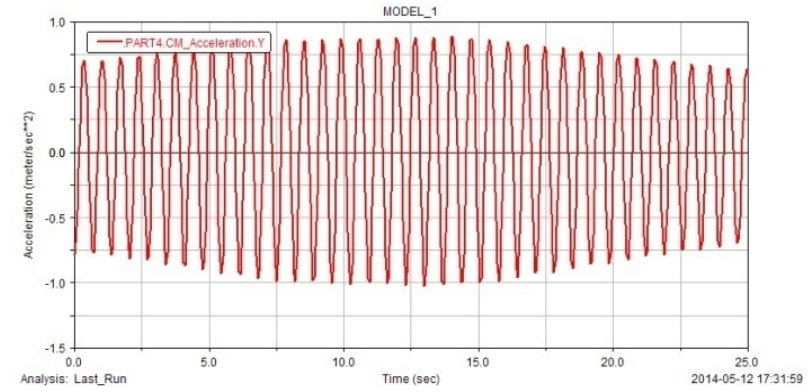

Fig. 10 the relationship between the acceleration and the time of the screen box in the $\mathrm{Y}$ direction

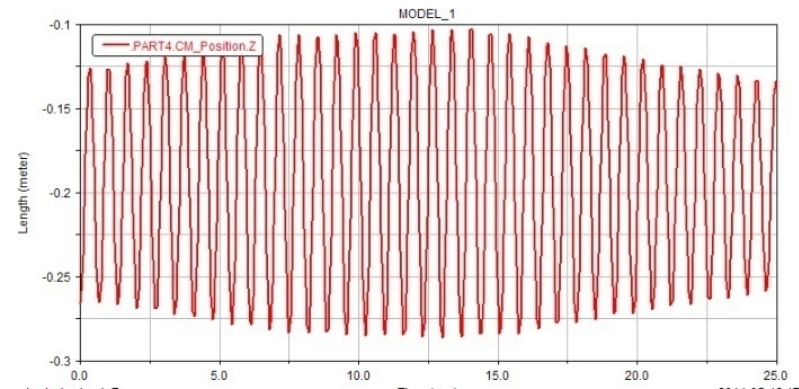

Fig.9 the relationship between the displacement and the time of the screen box in the $\mathrm{Z}$ direction

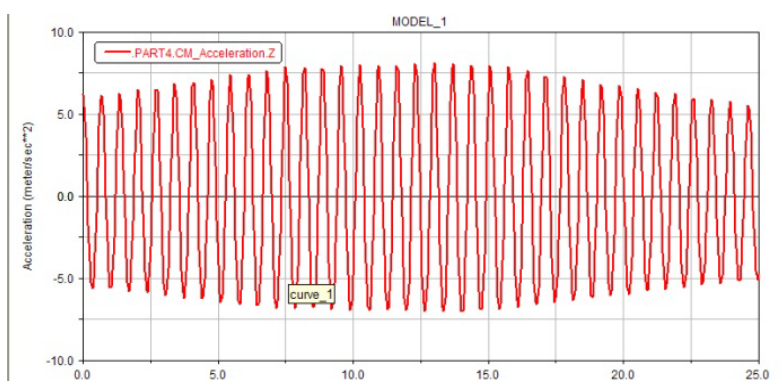

Fig.11 the relationship between the acceleration and the time of the screen box in the $\mathrm{Z}$ direction

From the above results, the box can be made in the direction of $\mathrm{Y}$, and in the direction of the $\mathrm{Z}$, it was a circular motion, meanwhile, the amplitude and acceleration of the vibration exciter in the $Y$ and $\mathrm{Z}$ directions were obtained at the given speed.

It can be seen that the motion of the screen box was changed according to a certain period Through the simulation of ADAMS and combined with the results, and the moving process was simple and controllable, and the maximum value of the acceleration and amplitude of the screen box in $\mathrm{Y}$ and $\mathrm{Z}$ directions was obtained. By analyzing the characteristic parameters such as frequency and amplitude of the circular vibrating screen, the screening performance can be obtained, which provides reference and basis for the following design and product improvement.

By throwing index to evaluate the performance of $\mathrm{K}$ circular vibrating screen,

$$
\mathrm{K}=\mathrm{Bn} / \mathrm{Gn}=\mathrm{AW} 2 \sin (\mathrm{a}+\mathrm{b}) / \mathrm{gcosb}
$$

Type: $\mathrm{Bn}$ - particles in the maximum acceleration perpendicular to the direction of the plate $(\mathrm{mm} / \mathrm{s} 2)$

A - two amplitude (mm)

a-The projection angle $\alpha\left({ }^{\circ}\right)$ In general 45

b-The screen angle $\beta\left({ }^{\circ}\right)$

g-Gravitational acceleration ( $\mathrm{mm} / \mathrm{s} 2)$

$\mathrm{K}$ in less than 1.5, throwing index low show that vibration effect was not obvious, indicating that material was not fully thrown up, and the screening efficiency was not high, easy to cause the blocking of the equipment.

\section{Conclusions}

The 3D model was established based on the full understanding of the structure principle of the circular vibrating screen, and by using the finite element software ANSYS, the modal analysis of the circular vibrating screen was carried out, and the natural frequency of the device was obtained. 
The frequency of the motor was far from its natural frequency (above 13\%) compared with the operating frequency, and the motor parameters are reasonable, which can avoid the resonance phenomenon. Using ADAMS software to simulate the kinematics of the model to determine the use of the speed of the machine can get a good screening effect, and provide reference and basis for the follow-up design and product improvement.

\section{Acknowledgements}

This work was financially supported by 2013 university student innovative undertaking training programs (Project No.201310361037) and the HuaiNan Science and technology projects (Project No. 2013A4008).

\section{References}

[1] Kang Zhang, Fei Geng. Development trend of coal screening equipment[J]. Mining machinery, 2010(24):11-13 .

[2] Nianqin Guo, Leping Luo. Finite element and modal analysis of ultra heavy vibrating screen box [J]. Mechanical and electrical engineering technology, 39, 2010 (04):32-34.

[3] Xin Du, Bo Chen. Current situation and trend of development of vibration screening machinery [J]. scientific and technical information, 109 (10): 2010

[4] Qian Chen. Working principle and parameters of vibrator offset type vibrating screen to set the [J]. color equipment, 1997 (4): 15 -21

[5] Chunyuan Cai. Mechanical parts design handbook (Third Edition) [M]. Beijing: Metallurgical Industry Press, 1996

[6] Lingqin Meng, Zhiwei Wang, Hongling Yuan, Linhai Bo. Linear vibrating screen structure vibration modal analysis [J]. Mining machinery, 2008 (9): 84 - 86.

[7] Nianqin Guo, Leping Luo. Finite element and modal characteristics analysis of [J]. mechanical and electrical engineering technology, 201034 (4) 32-37.

[8] ZhongJun Yin, Jianping Liu, Zhuandong Zhao. Under the action of alternating load large vibrating screen should force analysis [J]. Journal of Tianjin University, 2007 (11) 1363-1366. 